\title{
La eficiencia de la responsabilidad ampliada del productor en la gestión de aceites usados con diferenciación del producto*
}

\author{
ASUNCIÓN ARNER GÜERRE \\ Universidad de Zaragoza, Grupo Decisión Multicriterio de Zaragoza, C/ Doctor Cerrada 1-3, 50005 \\ Zaragoza.E-mail: aarner@unizar.es
}

\begin{abstract}
RESUMEN
El Real Decreto 679/2006, de aceites industriales usados, estableció la responsabilidad ampliada del productor (RAP) en la gestión de aceites usados. En 2015, la organización de la RAP se modifica con la entrada en vigor del Real Decreto 110/2015, sobre residuos de aparatos eléctricos y electrónicos (RAEE). En este contexto, los resultados de la evaluación de la eficiencia de la RAP difieren de los obtenidos previamente, por los que la RAP en la gestión del aceite usado constituye un estándar de material reciclado (EMR) aplicado al sector del lubricante. Desde la perspectiva de la gestión del aceite usado, la competencia entre distintos sistemas integrados de gestión por el aceite usado justifica que el resultado de la evaluación de la eficiencia sea indeterminado. Si se considera la evolución del mercado de lubricantes, se pone de manifiesto que una demanda de lubricantes más diferenciada es incompatible con el EMR.
\end{abstract}

Palabras clave: Responsabilidad ampliada del productor, Aceites usados, Eficiencia, Diferenciación del producto, Residuos Marpol.

\section{The Efficiency of Extended Producer Responsibility in Waste Oil Management with Product Differentiation}

\begin{abstract}
Royal Decree 679/2006, on used industrial oils, established the extended producer responsibility (EPR) in the management of used oils. In 2015, the organization of the EPR is modified with the entry into force of Royal Decree 110/2015, on waste electrical and electronic equipment (WEEE). In this context, the results of the evaluation of the efficiency of EPR differ from those previously obtained, by which the EPR in the management of used oil constitutes a standard of recycled material (SRM) applied for the whole sector of lubricants. From the perspective of waste oil management, the competition between different integrated management systems for waste oil justifies that the result of the evaluation of the efficiency is indeterminate. If the lubricants market is considered it is shown that a demand for more differentiated lubricants, is incompatible with the SRM.

Keywords: Extended Producer Responsibility, Waste Oils, Efficiency, Product Differentiation, MARPOL Waste Oils.
\end{abstract}

Clasificación JEL: H21, H23, Q53

\footnotetext{
* Agradezco los comentarios del revisor anónimo del artículo.
}

Artículo recibido en junio de 2018 y aceptado en septiembre de 2018

Artículo disponible en versión electrónica en la página www.revista-eea.net, ref. ə-36314 


\section{INTRODUCCIÓN}

Los aceites usados comprenden todos los aceites minerales o sintéticos, industriales o de lubricación, que han dejado de ser aptos para el uso originalmente previsto y constituyen un residuo peligroso ${ }^{1}$. La responsabilidad ampliada del productor (RAP) es una política particularmente útil en la gestión de aquellos residuos con legislación específica como embalajes, residuos de aparatos eléctricos y electrónicos (RAEE), baterías, neumáticos, vehículos fuera de uso (VFU) o aceites usados ${ }^{2}$. De acuerdo con Fleckinger y Glachant (2010), cualquier programa de RAP implica la responsabilidad del productor en la recogida y tratamiento de los residuos que genera su producto o la obligación legal de financiar dichas actividades. Frecuentemente, el regulador establece también objetivos de reciclaje. Los productores cumplen sus obligaciones, individualmente o creando, junto con otros productores, las que denominan organizaciones de responsabilidad del productor (ORP).

El Real Decreto 679, de 2 de junio de 2006, establece la RAP en la gestión de aceites usados, así como los objetivos de recuperación del 95\% de aceites usados sujetos al real decreto, el 1 de julio de 2006, y de regeneración del 55\% y 65\% de los aceites usados regenerables, respectivamente, el 1 de enero de 2007 y $2008^{3}$. En consecuencia, la financiación de la gestión de los aceites usados se efectúa a partir de la aportación del fabricante de lubricantes, de 0,06 €/kilogramo de aceite lubricante comercializado al sistema integrado de gestión de aceites usados

${ }^{1}$ Los aceites usados se reutilizan por el proceso de regeneración, proceso por el que se obtienen aceites base para la fabricación de lubricantes de los aceites usados, y combustión o valorización energética del aceite usado. La regeneración consiste en la eliminación de los contaminantes, productos de la oxidación y aditivos, que contienen los aceites usados, para la obtención de aceite lubricante base (Angulo y otros, 1996; Gómez-Miñana, 1993; LLobet Díaz, 1995 y Ramsden, 1995).

${ }^{2}$ La Directiva marco sobre residuos (Directiva 2008/98/EC) establece un marco legal común para la aplicación de la RAP, principio que constituye uno de los medios para apoyar el diseño y fabricación de bienes que tengan plenamente en cuenta y faciliten el uso eficaz de los recursos durante todo su ciclo de vida, incluidos su reparación, reutilización, desmontaje y reciclado sin perjudicar a la libre circulación de bienes en el mercado interior. En España, la trasposición de esta Directiva se efectuó por la Ley 22, de 28 de julio de 2011, relativa a los residuos y los suelos contaminados (B.O.E. de 29 de julio de 2011, núm. 181). En febrero de 2018, la Comisión de Medio Ambiente, Salud Pública y Seguridad Alimentaria del Parlamento Europeo alcanzó el acuerdo provisional sobre una nueva directiva marco de residuos para la UE, dentro del paquete de medidas para impulsar la transición de Europa hacia una economía circular "Cerrar el círculo: un plan de acción de la UE para la economía circular COM(2015) 614 final”, que en particular contemplan fomentar un mayor uso de los instrumentos económicos y establecer requisitos generales para los sistemas de responsabilidad ampliada del productor.

${ }^{3}$ B.O.E. de 3 de junio de 2006, núm. 132. Los aceites usados afectos al Real Decreto 679 son los aceites minerales usados de los motores de combustión y de los sistemas de transmisión, los de los lubricantes, los de turbinas y de los sistemas hidráulicos, así como las mezclas y emulsiones que los contengan y se excluyen los aceites marinos y de aviación, de proceso y grasas. 
(SIGAUS). En 2015, el Real Decreto 110/2015, sobre residuos de aparatos eléctricos y electrónicos (RAEE), por el que la responsabilidad del aceite usado contenido en los residuos de aparatos eléctricos y electrónicos se transfiere del productor de lubricantes a los fabricantes de dichos aparatos, modifica la organización de la RAP en la gestión de aceites usados ${ }^{4}$.

La literatura económica acerca de los programas de RAP alcanza actualmente cierto desarrollo. Runkel (2003) analiza, en competencia perfecta e imperfecta, la eficiencia de distintos instrumentos consistentes con la RAP, destacando la relación entre las características del producto (durabilidad del producto) y los costes de gestión de los residuos. Según este autor, la RAP incrementa la durabilidad del producto, pero también reduce el producto final que, en un contexto de competencia imperfecta, es previamente inferior al óptimo. Asimismo, Zink et al. (2017) en relación con los programas de reciclaje de los residuos al final de la vida útil de los productos señalan que el desplazamiento del uso de recursos naturales escasos por materiales reciclados no ocurre siempre en la misma proporción. En consecuencia, para obtener una medida cierta de los beneficios del reciclaje, debe conocerse el ratio de desplazamiento de materiales primarios y secundarios.

Fleckinger y Glachant (2010) profundizan en los resultados de Runkel (2003) al considerar endógeno al modelo el diseño de las OPR. Según estos autores, la calidad o diferenciación del producto influye en el coste de la gestión de los residuos que genere. Por consiguiente, la organización de un programa de RAP inevitablemente afecta a la competencia en el mercado y a la estrategia de mercado de las empresas. En consecuencia, las OPR entrañan un riesgo adicional de colusión y es necesario regular las tarifas que financian sus actividades. En este caso, un modelo que considere la diferenciación del producto constituye el instrumento adecuado para investigar la relación entre las características del producto y el coste de la gestión de los residuos generados.

Previamente, en un contexto de competencia perfecta, la literatura económica ha evaluado la eficiencia de distintos instrumentos económicos, al objeto de promover la recogida y reciclaje de los residuos, aplicados en la fase de producción -upstream- consistentes, en algunos casos, con los programas de RAP. Diversos estudios concluyen que el sistema de depósito-reembolso, por el que el productor soporta un impuesto sobre el producto y su destino es subvencionar el sector del reciclaje, es la política más eficiente para reducir la cantidad de residuos a eliminar porque reúne los dos efectos que caracterizan a un impuesto Pigouviano: la reducción del producto y la sustitución de recursos naturales escasos por materiales reciclados (Dinan, 1993; Fullerton y Kinnaman, 1995; Sigman, 1995; Palmer y Walls, 1997 y 1999 y Fullerton y Volverton, 2000).

\footnotetext{
${ }^{4}$ B.O.E. de 21 de febrero de 2015, núm. 45.
} 
Algunos estudios concluyen que esta política es consistente con la RAP (Palmer y Walls, 1999). De acuerdo con Tsai y otros (2013), incluso si el mercado es no competitivo, el sistema de depósito-reembolso es consistente con la RAP. Asimismo, los sistemas de retorno de los residuos, junto con el mandato de cumplir con un objetivo de reciclaje, es consistente con la RAP y la eficiencia relativa de estos dos instrumentos depende de la eficiencia de la tecnología de reciclaje.

El estándar de material reciclado (EMR) u obligación de que el producto incorpore un determinado porcentaje de material reciclado en su composición es, de acuerdo con Walls (2006), una política consistente con la RAP. Su aplicación en relación con la industria, mediante un sistema de permisos negociables -por el que aquellas empresas que utilizan inputs reciclados en exceso en relación con el estándar venden permisos a las que no lo cumplen- confiere flexibilidad y reduce el coste de esta política (Palmer y otros, 1995). De acuerdo con Sigman (1995), el EMR es eficiente si el número de permisos se establece en relación con el EMR y, en otro caso, el resultado es indeterminado y el coste mayor que el de un impuesto sobre el producto.

En este contexto, algunos autores han definido el diseño verde (green design) como el proceso en el que los atributos medioambientales son considerados con objetivos del diseño de los productos (Fullerton y Wu, 1998). Eichner y Pethig (2001) consideran el diseño del producto como el contenido en el producto de diferentes materiales. El contenido de un material en los residuos incrementa la productividad del reciclaje y favorece un diseño eficiente. Por consiguiente, los incentivos en precios transmitidos al productor pueden promover un diseño eficiente. Calcott y Walls (2002) consideran que, en ausencia de un comportamiento competitivo del mercado, el sistema de depósito-reembolso, junto con un impuesto a la eliminación final, promueve el diseño que garantiza un nivel suficiente de reciclaje. El diseño óptimo que maximice el reciclaje se obtiene únicamente si el mercado es eficiente.

La evaluación de la eficiencia de una subvención o un EMR para promover la regeneración de aceites usados se ha efectuado previamente de acuerdo con Arner y otros (2005, 2006). Posteriormente, la evaluación de la eficiencia de la RAP en la gestión de aceites usados ha puesto de manifiesto que SIGAUS constituye un EMR aplicado al sector del lubricante (Arner, 2017a) ${ }^{5}$. El precio del permiso en este mercado equivale a la aportación del fabricante de lubricantes a SIGAUS de 60€/tonelada y el porcentaje de aceite usado de origen

\footnotetext{
${ }^{5}$ El RD 679, de 2 de junio de 2006, se ha analizado también desde el punto de vista de los efectos dinámicos que los objetivos ecológicos establecidos ejercen en la cantidad de aceites usados destinados a regeneración y el precio de los aceites lubricantes base (Arner, 2017b), sobre la base de los estudios que analizan el efecto de los shocks petrolíferos sobre el comportamiento de la macroeconomía (veáse, por ejemplo, Maslyuk y Dharmaratna, 2013).
} 
desconocido, denominado free riders, que asume voluntariamente, del 1,8\%. Desde 2015, el Real Decreto 110/2015 ha modificado la organización de la RAP en la gestión de aceites usados. El objetivo de este trabajo es evaluar la eficiencia de la RAP en la gestión de aceites usados cuando la RAP se aplica a los RAEE. La metodología consiste en el cálculo de las elasticidades de la función de oferta y demanda de aceites usados destinados a regeneración y su aplicación en la evaluación de la eficiencia de la RAP efectuada previamente.

En este caso, los resultados difieren de los obtenidos previamente, por los que la RAP en la gestión de aceites usados constituye un EMR aplicado al sector del lubricante. Desde la perspectiva de la gestión del residuo, la competencia entre distintos sistemas integrados de gestión (SIGs) por el aceite usado implica que el EMR no esté establecido a priori y el resultado sea indeterminado. Si se considera la evolución del mercado de lubricantes, los resultados se justifican porque una demanda de lubricantes más diferenciada -de aceites sintéticos y semisintéticosresulta incompatible con el EMR. El trabajo se estructura en cuatro secciones. A continuación, se presentan las principales características de la gestión de aceites usados en España. El tercer apartado se dedica a la estimación de una función de oferta y demanda de aceites usados destinados a regeneración para el cálculo de las elasticidades. El cuarto apartado presenta la evaluación de la eficiencia de la RAP. Un último apartado recoge las principales conclusiones del trabajo.

\section{LA GESTIÓN DE LOS ACEITES INDUSTRIALES USADOS EN ESPAÑA}

El R.D. 679/2006, de aceites industriales usados, por el que se establece la responsabilidad del productor en la gestión de este residuo, permite distinguir dos políticas de gestión de aceites usados en España, diferentes en sus objetivos y los instrumentos aplicados. La primera de estas políticas, de aplicación desde la liberalización del mercado de lubricantes en enero de 1989 y hasta la entrada en vigor del R.D. 679/2006, se caracteriza en sus objetivos por el aumento de la tasa de recogida del residuo y, en sus instrumentos, por la autorización de la combustión -como proceso de reutilización del aceite usado junto con el de regeneración- y por la concesión de subvenciones a la recogida y reutilización del residuo. Desde mediados de los noventa, la exención en el Impuesto sobre hidrocarburos de los aceites usados utilizados como combustible promueve, a su vez, la valorización energética del aceite usado con producción de electricidad. El resultado de esta política fue la ampliación de la capacidad de recogida del residuo, siendo el destino principal del aceite usado la combustión, y el aumento de la tasa de recogida, que al final de la década es del $86,7 \%$ de los aceites usados generados. La Directiva 76/2000/CE, sobre incineración de residuos, tuvo gran incidencia en el modelo anterior favoreciendo el destino de los aceites 
usados a regeneración, en detrimento de la combustión, que alcanza en 2005 el $65 \%$ de los aceites usados recogidos ${ }^{6}$.

La segunda política hace referencia a la aplicación del Real Decreto 679/2006 por el que se transfiere al fabricante de lubricantes la responsabilidad financiera de la gestión de los aceites usados generados por el consumo de su producto. Los fabricantes de lubricantes dieron respuesta a este mandato, de forma colectiva, constituyendo SIGAUS. La responsabilidad financiera de SIGAUS, en 2007 y 2008, fue del $88,49 \%$ de los aceites usados sujetos al real decreto y SIGAUS asumió la financiación de un porcentaje de aceite usado de origen desconocido, del 1,8\%. Posteriormente, los sucesivos estudios de mercado han modificado estos porcentajes (SIGAUS, 2013) ${ }^{7}$. En 2012, la responsabilidad financiera de SIGAUS fue del $87,87 \%$ y asumió de forma voluntaria el 2,12\% del aceite sujeto al real decreto. En 2013, estos porcentajes fueron, respectivamente, 87,13\% y $2,89 \%$. Por consiguiente, la responsabilidad financiera final, previamente a aplicar la RAP en la gestión de otros residuos que contienen aceites usados, es del 90,02\% del aceite usado sujeto al Real Decreto 679/2006.

En 2015, con la entrada en vigor del Real Decreto 110/2015, la responsabilidad financiera de SIGAUS se establece en el 87,15\%, mientras que el aceite que asume voluntariamente es del $1,41 \%$, siendo la responsabilidad financiera final del 88,56\% del aceite usado sujeto a RAP (SIGAUS, 2016a). En 2016, en un contexto de precios bajos del petróleo, el sistema de financiación se modifica, a su vez, al establecer SIGAUS una financiación adicional indexada a la cotización internacional de los lubricantes (SIGAUS, 2016b). La responsabilidad financiera de SIGAUS, con la entrada en vigor el Real Decreto 20/2017, de vehículos fuera de uso, por el que se transfiere la responsabilidad de la gestión de los aceites usados contenidos en los residuos de los vehículos automóviles a los fabricantes de los mismos, es del $85,34 \%$ y la bolsa de fraude que asume voluntariamente del $1,23 \%$, siendo la responsabilidad financiera final de SIGAUS del $86,57 \%$ del aceite industrial usado sujeto a RAP (SIGAUS, 2018) ${ }^{8}$. El resto del mercado, sin exportación, que alcanza la cifra de 343.430 toneladas de aceites industriales sujetos a RAP en 2017, corresponde en el 6,82\% al aceite adherido al SIG de productores independientes (SIGPI), el 0,72\% a importación de vehículos, el $0,63 \%$ a RAEE y el $4,95 \%$ a $\mathrm{VFU}^{9}$. En su conjunto, los aceites industriales

\footnotetext{
${ }^{6}$ Ley de 5 de junio de 2013, traspone a la legislación nacional la Directiva 2010/75/EU, relativa a las emisiones industriales, por la que se deroga la Directiva 2000/76/EC13 (B.O.E. de 12 de junio de 2013, núm. 140).

${ }^{7}$ Estudios de mercado efectuados por la consultora independiente PwC.

${ }^{8}$ B.O.E. de 21 de enero de 2017, núm. 18.

${ }^{9}$ Los vehículos importados se agrupan mayoritariamente en la Asociación Nacional de Importadores de Automóviles, Camiones, Autobuses y Motocicletas (ANIACAM) y la Asociación Nacional de Fabricantes de Automóviles (ANFAC).
} 
comercializados en España y sometidos a RAP representan un 82\% del mercado total, siendo el $18 \%$ restante el correspondiente a grasas, aceites para marina y aviación y aceites de proceso.

Simultáneamente, desde 2013, ha tenido lugar una serie de cambios en la fiscalidad medioambiental, en el ámbito de la Ley de Impuestos Especiales, tendentes a incentivar la valorización energética del aceite usado ${ }^{10}$. En 2013, en el Impuesto sobre hidrocarburos, se establece el tipo impositivo de $14 €$ por tonelada de aceite lubricante puesto en el mercado, que desde 1993 era cero ${ }^{11}$. En 2014, se establece la exención en el Impuesto sobre la electricidad de la energía eléctrica consumida por los titulares de las instalaciones de producción de energía eléctrica acogidas al régimen específico de producción de electricidad (Artículo 94.5 Ley de Impuestos Especiales) ${ }^{12}$. En relación con esta actividad, de acuerdo con el Real Decreto 413/2014, de 6 de junio, por el que se regula la actividad de producción de energía eléctrica a partir de fuentes de energía renovables, cogeneración y residuos y la Orden IET/1045/2014, de 16 de junio, por la que se establece el régimen retributivo de las instalaciones de régimen específico, en los ejercicios de 2016, 2017 y 2018, se han actualizado los correspondientes parámetros retributivos.

En el ámbito más específico de los residuos MARPOL tipo c (residuos de sentinas de cámara de máquinas o de equipos de depuración de combustible y aceite de motores), no sujetas al Real Decreto 679, la Ley de Impuestos Especiales contempla la exención, en el Impuesto sobre hidrocarburos, de la fabricación e importación de los carburantes utilizados en la navegación en aguas comunitarias (Art. 51.2.b. Ley de Impuestos Especiales). La reforma en 2014 de esta Ley establece, a su vez, la exención en el Impuesto sobre la electricidad de la producción de electricidad a bordo de las embarcaciones (Art. 94.7 Ley de Impuestos especiales).

De acuerdo con la Figura 1, los incentivos a la valorización energética de los aceites usados han supuesto el aumento de la cantidad de aceites usados

\footnotetext{
${ }^{10}$ Ley 38/1992, de 28 de diciembre, de Impuestos Especiales (B.O.E. de 29 de diciembre de 1992, núm. 312).

${ }^{11}$ Ley 16/2013, de 29 de octubre, por la que se establecen determinadas medidas en materia de fiscalidad medioambiental y se adoptan otras medidas tributarias y financieras (B.O.E. de 30 de octubre de 2013, núm. 260). El gravamen de los aceites lubricantes, en el Impuesto sobre hidrocarburos se eliminó en 1993 al objeto de favorecer que los productores establecieran un sistema para la financiación de la gestión de los aceites usados (MOPTMA, 1993).

${ }^{12}$ Ley 28/2014, de 27 de noviembre, por la que se modifican la Ley 37/1992, de 28 de diciembre, del Impuesto sobre el Valor Añadido, la Ley 20/1991, de 7 de junio, de modificación de los aspectos fiscales del Régimen Económico Fiscal de Canarias, la Ley 38/1992, de 28 de diciembre, de Impuestos Especiales, y la Ley 16/2013, de 29 de octubre, por la que se establecen determinadas medidas en materia de fiscalidad medioambiental y se adoptan otras medidas tributarias y financieras.
} 
destinados a regeneración y combustión desde 2015. Asimismo, desde 2013 se ha recuperado el aceite industrial puesto en el mercado (Figura 2). En este mercado, de acuerdo con ASELUBE (2018), los productos sintéticos y semisintéticos, aceites lubricantes de aceite base de origen no mineral, constituyen actualmente el 92,8\% del total del mercado de lubricantes en 2017.

Figura 1

Evolución de las cantidades de aceites usados sometidas a distintos tratamientos (toneladas)

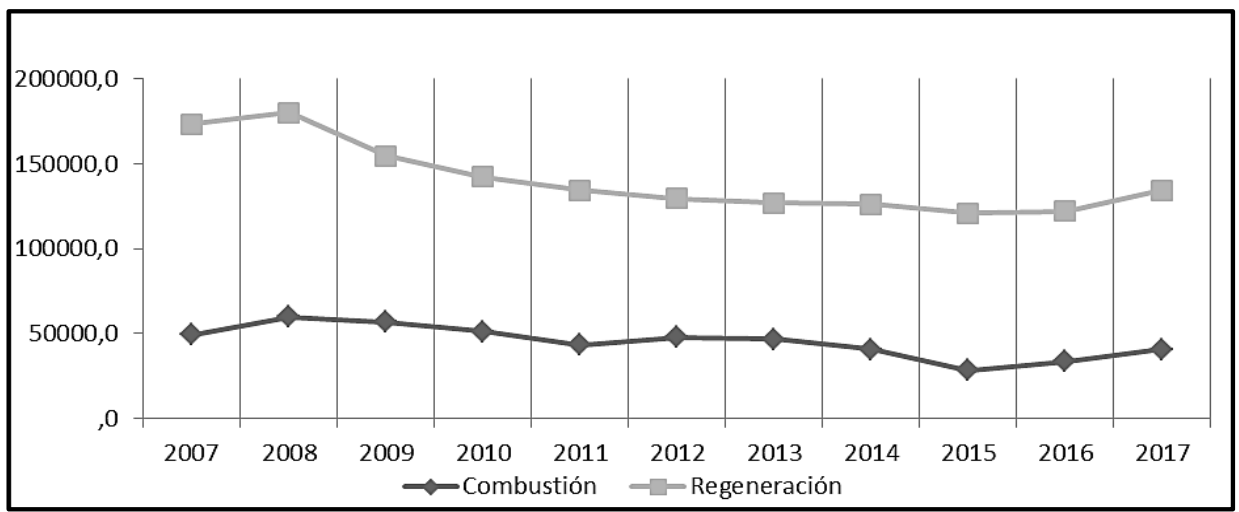

Fuente: SIGAUS (2018a).

Figura 2

Evolución del aceite industrial puesto en el mercado (toneladas)

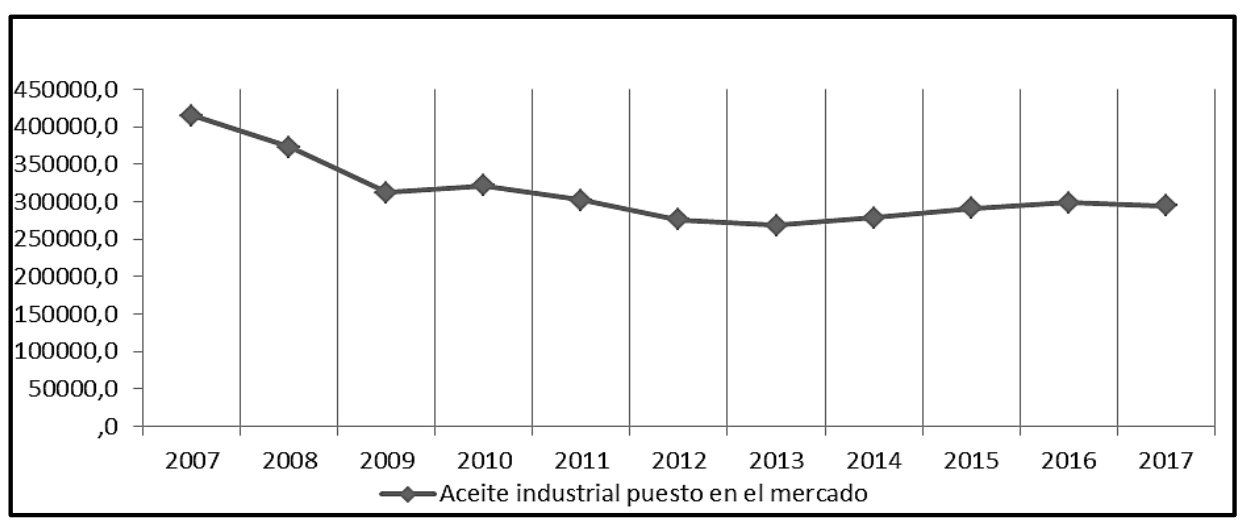

Fuente: SIGAUS (2018a).

Actualmente, la Orden APM/205/2018, de 22 de febrero, establece los criterios para determinar cuándo al aceite usado procesado procedente del tratamiento de aceites usados para su uso como combustible deja de ser residuo 
con arreglo a la Ley 22/2011, de 28 de julio, de residuos y suelos contaminados ${ }^{13}$. En un ámbito más específico, la Orden APM/206/2018, de 22 de febrero, establece estos criterios en referencia al uso en buques del fuel recuperado procedente del tratamiento de residuos MARPOL tipo c para su uso como combustible en buques ${ }^{14}$. El productor demostrará que cumple dichos criterios aplicando un sistema de gestión de calidad verificado externamente, como la norma ISO 9001 u otra similar. Ambas regulaciones, que las empresas de transformación del aceite usado deberán cumplir con carácter obligatorio desde 2020, tienen por objeto facilitar la comercialización de los productos resultantes, aceites usados para su uso como combustible en todo tipo de instalaciones y el fuel recuperado de los residuos MARPOL tipo c para su uso en buques, sin que deban someterse al régimen jurídico de los residuos en dichos intercambios en territorio nacional ${ }^{15}$.

\section{ESTIMACIÓN DE UNA FUNCIÓN DE OFERTA Y DEMANDA DE ACEITES USADOS DESTINADOS A REGENERACIÓN}

La estimación de una función de oferta y demanda de aceites usados destinados a regeneración se fundamenta en el proceso de generación y regeneración del aceite usado en SIGAUS (Figura 3). En el mercado de aceites lubricantes, la producción y el consumo de los aceites base se adiciona con el porcentaje, $\gamma$, de aditivos incorporados para la fabricación de lubricantes, para determinar la producción y el consumo de aceites lubricantes. La participación de los aditivos en la composición de los lubricantes ha evolucionado con la diferenciación y el destino final de los lubricantes hasta ser, actualmente, del $20 \%$.

Si se aplica la RAP en la gestión de los aceites usados, el porcentaje de generación de aceites usados a partir del consumo de aceites lubricantes, $\alpha$, se modifica en cada ejercicio. En el período 2006-2017, el coeficiente $\alpha$ se sitúa entre el $41,4 \%$ y el $45,57 \%{ }^{16}$. A su vez, los aceites usados se destinan a regeneración en un porcentaje variable, $\mu$, que en SIGAUS es igual o superior al

\footnotetext{
${ }^{13}$ B.O.E. de 2 de marzo de 2018, núm. 54. De acuerdo con el artículo 5 de la Ley 22/2011, de 28 de julio, los criterios que deben cumplir determinados residuos para dejar de considerarse como residuos son: los productos resultantes deberán usarse habitualmente para finalidades específicas; existirá un mercado o una demanda para los mismos; deberán cumplir los requisitos técnicos para finalidades específicas, la legislación existente y las normas aplicables a los productos y su uso no generará impactos adversos para el medio ambiente o la salud.

${ }^{14}$ B.O.E. de 2 de marzo de 2018, núm. 54.

${ }^{15}$ La Comisión Europea ha manifestado que los criterios nacionales de la condición de fin de residuo sólo serán vinculantes dentro del Estado miembro que los ha establecido.

${ }^{16}$ SIGAUS (2018).
} 
65\%. En consecuencia, el precio de los aceites usados se determina, principalmente, en relación con el precio de los aceites de primer refino.

Figura 3

Aceite usado generado (AUG) y aceite base regenerado (ABR) en SIGAUS

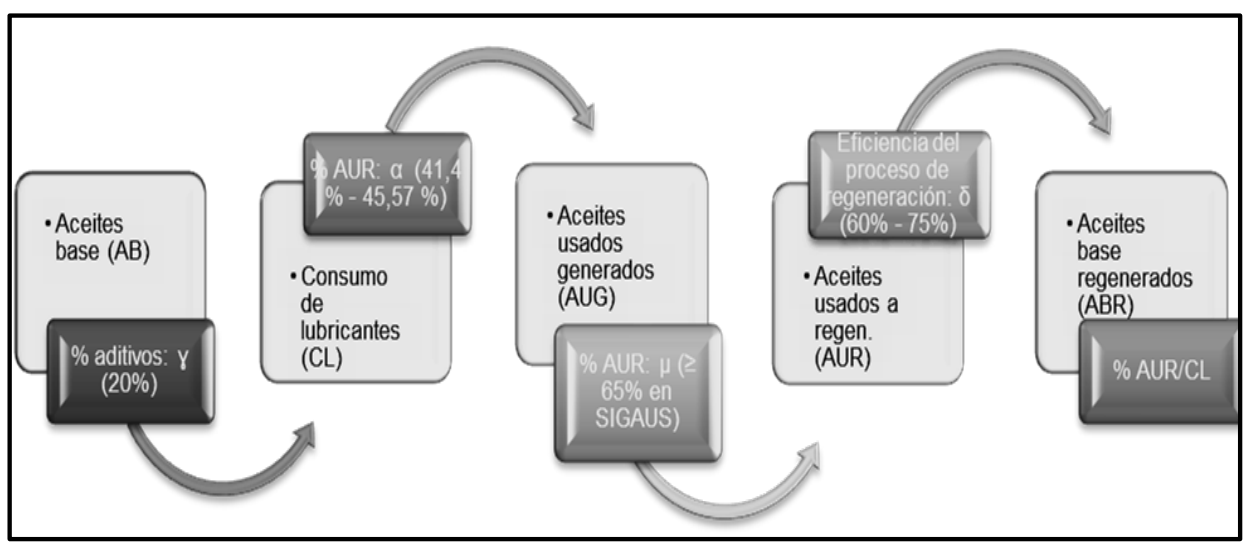

Fuente: Elaboración propia.

Las variables utilizadas en la estimación de una función de oferta y demanda de aceites usados destinados a regeneración son la variable cantidad de aceites usados destinados a regeneración, AUR, y la variable precio de los aceites base de primer refino, $P P R$, expresado en unidades monetarias corrientes. El período muestral es 1964-2016 y los datos se refieren a períodos anuales ${ }^{17}$. Actualmente, se han producido cambios de relevancia en la organización de la RAP, en la gestión de aceites usados, con la entrada en vigor del Real Decreto 110/2015. En consecuencia, el análisis del orden de integración de las variables se efectúa asumiendo la posible existencia de cambios estructurales en el

${ }^{17}$ Los datos de la variable, AUR, previamente a 1993, se obtienen de las Memorias de la Delegación del Gobierno en CAMPSA (varios años), de las Memorias de CAMPSA (varios años) y de la Resolución ministerial de la concesión de subvenciones en cada ejercicio. En 1993, la información es facilitada por la Consejería de Medio Ambiente de la Comunidad Valenciana (comunicación personal). Desde 1994, la información procede del Ministerio de Medio Ambiente (comunicación personal) y de la publicación Medio Ambiente en España (2006). El factor de conversión de aceites usados en aceites regenerados utilizado es de 0,6. La variable, $P P R$, es el precio de los aceites lubricantes base de primer refino en términos nominales. En la etapa del Monopolio de Petróleos, los datos proceden de las Memorias de la Delegación del Gobierno en CAMPSA (varios años). En el período de 1987-1991 son precios de mercado proporcionados por REPSOL (comunicación personal). Desde 1992, son precios de importación obtenidos a partir de las bases de datos de comercio exterior DATACOMEX (Ministerio de Industria y Tecnología), que puede consultarse en http://datacomex.comercio.es/ [August 24, 2017], y STACOM (ICEX), disponible en http://estacom.icex.es/ [August 24, 2017]. 
comportamiento de las variables, aplicando el test de raíces unitarias con ruptura estructural.

Asimismo, de acuerdo con la Figura 3, los aceites usados generados después del consumo de los lubricantes se reutilizan en la fabricación de nuevos lubricantes. Por consiguiente, en la estimación de la función de oferta y demanda, para el cálculo de las elasticidades, se considera que la cantidad de aceites usados destinados a regeneración depende de su valor en ejercicios anteriores. La estimación de la función de oferta y demanda se efectúa de forma individual, debido que la variable precio, $P P R$, es una variable exógena en el mercado de los aceites usados destinados a regeneración. En definitiva, en ambas funciones, se estima una regresión de cointegración entre $A U R$ y $P P R$, utilizando el estimador de Mínimos Cuadrados Ordinarios Dinámicos (MCOD).

\subsection{Análisis del orden de integración de las variables}

El análisis del orden de integración de las variables AUR y $P P R$ se realiza, en esta ocasión, aplicando el test de raíces unitarias con ruptura estructural, por el que se selecciona la ruptura estructural minimizando el estadístico $t$ de Dickey-Fuller ${ }^{18}$. En la especificación de la regresión, así como en la ruptura estructural, se ha considerado únicamente un término constante y en la determinación del orden de los retardos el criterio de información Akaike, con un número máximo de retardos igual a 10. El cambio estructural propuesto es del tipo innovación particular o aislada. La elección del modelo en niveles para la determinación del orden de integración de las variables se ha realizado a partir de la estimación de la ecuación del test Dickey-Fuller Aumentado (DFA) ${ }^{19}$.

Tabla 1

Test de raíz unitaria con ruptura estructural*

\begin{tabular}{|cccccc|}
\hline \multicolumn{1}{|c}{ Variable } & \multicolumn{3}{c|}{ AUR } & PPR \\
\hline & & t-estadístico & Probabilidad & t-estadístico & Probabilidad \\
\hline & & -5.797224 & $<0.01$ & -4.262351 & 0.0819 \\
\hline \multirow{4}{*}{ Valores críticos } & Nivel & & & & \\
& significatividad & & & -4.949133 & \\
& $1 \%$ & -4.949133 & & -4.443649 & \\
& $5 \%$ & -4.443649 & & -4.193627 & \\
\hline
\end{tabular}

* Probabilidades asimptóticas (Vogelsang, 1993).

Fuente: Elaboración propia.

Los resultados del análisis del orden de integración, siendo la hipótesis nula, Ho, la existencia de una raíz unitaria en la variable, muestran que, al nivel de

\footnotetext{
${ }^{18}$ El estadístico $t$ del test de Dickey-Fuller puede verse en Maddala, G. y Kim, I. (1999).

${ }^{19}$ Véase, por ejemplo, Novales, A. (2010).
} 
significación del 5\%, la variable AUR es estacionaria. A su vez, al nivel de significación del $10 \%$, se acepta que la variable $P P R$ es estacionaria. Por consiguiente, se acepta la coherencia global del modelo en una estimación MCO. Finalmente, los resultados muestran un cambio estructural para la variable $A U R$ en 2000 y para la variable $P P R$ en 1991.

De acuerdo con los resultados de la estimación de la ecuación del test DFA, el coeficiente de la variable ficticia, por el que se reconoce un cambio estructural en 2000 en la variable AUR, es significativo al nivel de significación del $10 \%$. Sin embargo, el coeficiente de la variable ficticia, por el que se reconoce un cambio estructural en 1991 en la variable $P P R$, no es significativo a ningún nivel de significación. Consecuentemente, en la estimación de la función de oferta y demanda de aceites usados destinados a regeneración se considera únicamente el cambio estructural en 2000.

\subsection{Estimación de la función de oferta y demanda de aceites usados destinados a regeneración}

\subsubsection{Estimación de la función de oferta}

La función de oferta de aceites usados destinados a regeneración propuesta, si L es la notación logarítmica de la variable, es:

$$
L A U R=f(L P P R)
$$

Siendo el signo esperado del coeficiente de la variable $L P P R$ positivo.

De acuerdo con el test de raíces unitarias con ruptura estructural, en la estimación de la función de oferta de aceites usados destinados a regeneración se considera a priori la existencia de la ruptura estructural en la variable AUR en 2000, asociada con la normativa europea sobre incineración de residuos aprobada en dicho año. En consecuencia, se introduce una variable ficticia de carácter multiplicativo respecto de la variable precio, $D L P P R$, con valor 1 en 2000. Finalmente, la función de oferta de aceites usados destinados a regeneración a estimar es:

$$
L A U R_{t}=\alpha_{1}+\alpha_{2} L P P R_{t}+\alpha_{3} D L P P R_{t}+\varepsilon t
$$

Siendo $\varepsilon_{t}$ un término de error ruido blanco.

La Tabla 2 resume los resultados de la estimación utilizando el criterio de información Akaike (AIC) para la determinación del número de ventajas y retardos.

La estimación de la función de oferta de aceites usados destinados a regeneración pone de manifiesto que la inclusión de una variable ficticia que refleje la existencia de una ruptura estructural en el año 2000 no resulta significativa en la estimación MCOD, ni en una estimación MCO. Por consiguiente, puede afirmarse que la estimación por MCOD no presenta 
problemas de ruptura estructural y los resultados de la Tabla 2 omiten la inclusión de la variable ficticia $D L P P R$. Dichos resultados son satisfactorios en relación con la capacidad explicativa del modelo $\left(\mathrm{R}^{2}=0,75\right)$. Asimismo, se acepta la significatividad individual del coeficiente $\alpha_{1}$, igual a 4,92 y de la significatividad individual y el signo esperado del coeficiente $\alpha_{2}$, igual a 0,86 , al nivel de significación del 5\%.

\section{Tabla 2}

Estimación de la función de oferta de aceites usados destinados a regeneración

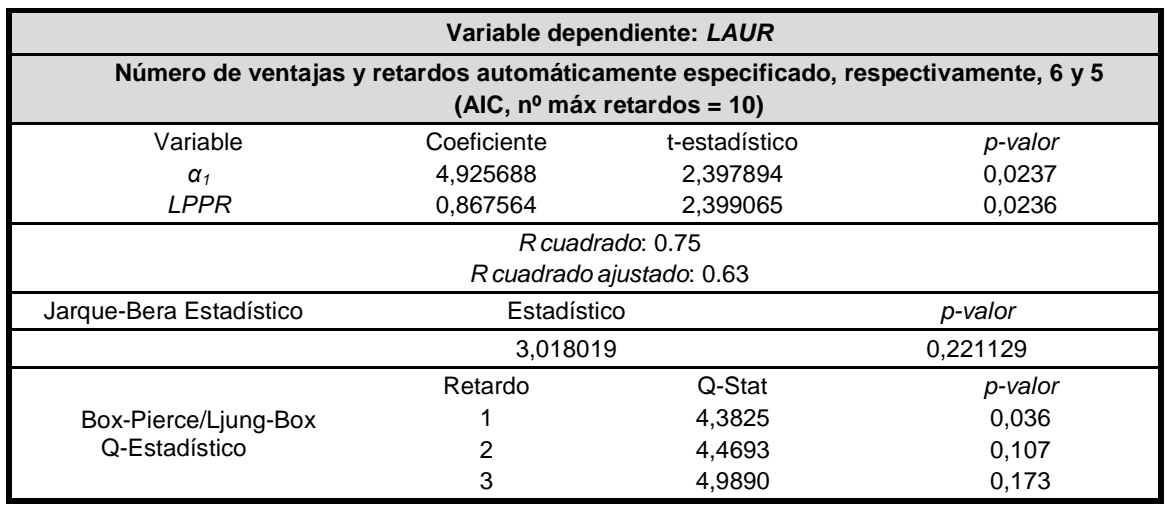

Fuente: Elaboración propia.

En relación con las características del término de error, según el estadístico de Jarque-Bera, se acepta la hipótesis nula de que los residuos se distribuyen según una distribución normal multivariante, al nivel de significación del 5\%. A su vez, según el estadístico Q se acepta la hipótesis nula de ausencia de autocorrelación en el segundo retardo, al nivel de significación del 5\%. En consecuencia, los resultados son suficientemente satisfactorios desde el punto de vista de la coherencia de la estructura estocástica de las variables y de la estimación, aunque existe autocorrelación en el término de error, siendo la elasticidad precio de la función de oferta de aceites usados destinados a regeneración, correspondiente al coeficiente $\alpha_{2}$, de signo positivo como se esperaba, es 0,86 .

\subsubsection{Estimación de la función de demanda}

En la estimación de la demanda, se propone la estimación de la función inversa de demanda de aceites usados destinados a regeneración al objeto de reflejar la ruptura estructural en el año 2000, asociada al cambio de la normativa europea de incineración de residuos. En consecuencia, si L es la notación logarítmica de la variable:

$$
L P P R=f(L A U R)
$$

Siendo el signo esperado del coeficiente de la variable $L A U R$ positivo. 
Finalmente, introduciendo una variable ficticia de carácter multiplicativo, con valor 1 en 2000, en relación con la variable cantidad, DLAUR, la función de demanda de aceites usados destinados a regeneración a estimar es:

$$
L P P R_{t}=\beta_{1}+\beta_{2} L A U R_{t}+\beta_{3} D L A U R_{t}+\mu_{t}
$$

Siendo $\mathrm{u}_{\mathrm{t}}$ un término de error ruido blanco.

La Tabla 3 resume los resultados de la estimación introduciendo, adicionalmente, una tendencia lineal. En este caso, el número de datos es insuficiente para la especificación automática del número de ventajas y retardos. En consecuenca, se ha supuesto un número de retardos y ventajas igual, respectivamente, a siete y cero ${ }^{20}$.

Tabla 3

Estimación de la función de demanda de aceites usados destinados a regeneración

\begin{tabular}{|c|c|c|c|}
\hline \multicolumn{4}{|c|}{ Variable dependiente: $L P P R$} \\
\hline \multicolumn{4}{|c|}{ Número ventajas y retardos es, respectivamente, 0 y 7 ( $n^{\circ}$ fijado) } \\
\hline Variable & Coeficiente & t-estadístico & $p$-valor \\
\hline$\beta_{1}$ & $-7,730857$ & $-2,997220$ & 0,0061 \\
\hline LAUR & 1,173671 & 4,712280 & 0,0002 \\
\hline DLAUR & $-0,228901$ & $-4,435490$ & 0,0002 \\
\hline @Tendencia & 0,069327 & 4,911033 & 0,0000 \\
\hline \multicolumn{4}{|c|}{$\begin{array}{c}R \text {-cuadrado: } 0.90 \\
R \text {-cuadrado ajustado: } 0.83\end{array}$} \\
\hline \multirow[t]{2}{*}{ Jarque-Bera Estadístico } & \multicolumn{2}{|c|}{ Estadístico } & $p$-valor \\
\hline & \multicolumn{2}{|c|}{3,092887} & \\
\hline \multirow{5}{*}{$\begin{array}{c}\text { Box- } \\
\text { Pierce/Ljung-Box } \\
\text { Q-Estadístico }\end{array}$} & Lag & Q-Stat & $p$-valor \\
\hline & 1 & 6,7694 & 0,009 \\
\hline & 2 & 7,9207 & 0,019 \\
\hline & 3 & 8,0805 & 0,044 \\
\hline & 4 & 8,6509 & 0,070 \\
\hline
\end{tabular}

Fuente: Elaboración propia.

Los resultados de la estimación son satisfactorios en relación con la capacidad explicativa del modelo $\left(\mathrm{R}^{2}=0,90\right)$ y la significatividad individual de todos los regresores (Tabla 3). En particular, el coeficiente $\beta_{2}$, igual a 1,17, es significativo al nivel de significación del $5 \%$. A su vez, el coeficiente $\beta_{3}$, igual a $-0,22$, es significativo al nivel de significación del $5 \%$. Por consiguiente, el signo del coeficiente de la variable $L A U R$ es el esperado.

En relación con las características del término de error, de acuerdo con el estadístico de Jarque-Bera, se acepta la hipótesis nula de que los residuos se distribuyen según una distribución normal multivariante, al nivel de significación

${ }^{20}$ Este resultado es el que automáticamente ofrece la estimación sin considerar la variable ficticia ni la tendencia lineal, aunque en dicha estimación los residuos presentan autocorrelación. 
del 5\%. Según el estadístico Q se acepta la hipótesis nula de ausencia de autocorrelación en el cuarto retardo, al nivel de significación del 5\%. En definitiva, los resultados son aceptables desde el punto de vista de la coherencia de la estructura estocástica de las variables y del ajuste de la estimación, aunque el término de error presenta autocorrelación. Por consiguiente, puede afirmarse que la elasticidad de LPPR (función de demanda) con respecto a la variable LAUR es 1,17 hasta 2000, mientras que, desde ese año, la función de demanda es inelástica, con un valor de la elasticidad respecto de la variable LAUR, de 0,94.

\subsubsection{Comparación de resultados}

Previamente, se efectuó el cálculo de las elasticidades de la función de oferta y demanda de aceites usados destinados a regeneración, aunque sin considerar la existencia de rupturas estructurales (Arner, 2017a). En este trabajo, la ampliación del período de estimación ha supuesto introducir en la estimación de una función de demanda de aceites usados destinados a regeneración una ruptura estructural, en el año 2000, en relación con el cambio en la normativa europea sobre incineración de residuos. En la Tabla 4, se presenta la comparación con los resultados obtenidos en ambos trabajos.

Tabla 4

Análisis empírico del mercado de aceites usados

\begin{tabular}{|ccccc|}
\hline RAP & $\begin{array}{c}\text { Período de } \\
\text { estimación }\end{array}$ & Estimador & $\begin{array}{c}\text { Elasticidad de la } \\
\text { función de oferta }\end{array}$ & $\begin{array}{c}\text { Elasticidad de la } \\
\text { función de demanda }\end{array}$ \\
\hline RAP (aceites usados) & $1964-2015$ & MCOD & 1,82 & 0,55 \\
\hline RAP (aceites usados y RAEE) & $1964-2016$ & MCOD & 0,86 & $\begin{array}{c}1,17 \\
0,94 \text { (desde 2000) }\end{array}$ \\
\hline
\end{tabular}

Fuente: Elaboración propia.

Según la Tabla 4, la aplicación de la RAP a los RAEE y la consiguiente reducción de la responsabilidad financiera de SIGAUS en la gestión del aceite usado sujeto a RAP, ha supuesto que la elasticidad precio de la función de oferta de aceites usados destinados a regeneración disminuya de 1,82 a 0,86. Asimismo, en la función de demanda, la relación entre la variable precio y la variable cantidad aumenta, desde 2000 , de 0,55 a 0,94 . Por consiguiente, el nuevo reparto de responsabilidades implica, en este caso, que la influencia de $L A U R$ en $L P P R$ aumenta.

\section{EVALUACIÓN DE LA EFICIENCIA DE LA RAP}

Previamente, sobre la base de Arner y otros (2005, 2006), la evaluación de la eficiencia de la RAP ha puesto de manifiesto que la RAP en la gestión de aceites usados constituye un EMR aplicado al sector del lubricante (Arner, 
2017a ${ }^{21}$. El análisis consiste en la determinación del precio del permiso y la aportación del productor al SIGAUS, calculando la incidencia marginal del precio del permiso y el coste privado marginal (CPM) y considerando que el legislador, mediante el Real Decreto 679/2006, establece un $d r^{*}$ igual al $21,95 \%{ }^{22}$. El precio del permiso, $\pi$, se obtiene a partir de, d $\pi$, igual a $21,95 \%$, con un nivel de intervención previo igual a cero, y la conversión en unidades monetarias (euros). Siendo la incidencia marginal del precio del permiso sobre el precio de oferta, $\mathrm{d} P \mathrm{~s} / \mathrm{d} \pi$, de $-2,14 \%$, el precio del permiso, $\pi$, es de $61,64 €$. En consecuencia, con un porcentaje de error del 0,10, los resultados ponen de manifiesto que la aportación del fabricante de lubricantes a SIGAUS es de $60 € /$ tonelada y la bolsa de fraude que asume voluntariamente, del 1,8\%. A su vez, el valor de la subvención que reciben los regeneradores por tonelada de aceite usado destinado a regeneración, siendo el coeficiente de generación de aceite usados a partir de aceites lubricantes de 0,4 , es de $180 €$ por tonelada. Finalmente, el CPM de esta política, de 1,25, es inferior al de una subvención, de 1,55 .

Considerando las elasticidades de oferta y demanda de aceites usados destinados a regeneración obtenidas en este trabajo, respectivamente, de 0,86 y 0,94 , el valor de la incidencia marginal del precio del permiso sobre $P s, \mathrm{~d} P \mathrm{~s} / \mathrm{d} \pi$, es positiva de 0,58. En este caso, siendo el precio del permiso, $\pi$, de 2,24, la aportación del fabricante se corresponde, casi en su totalidad, con el porcentaje de free riders que asume actualmente SIGAUS, de 1,23. En consecuencia, la evaluación de la eficiencia de la RAP difiere de los resultados obtenidos previamente, por los que la RAP en la gestión de aceites usados constituye un EMR aplicado al sector del lubricante. Desde la perspectiva de la gestión del aceite usado, este resultado se justifica porque el Real Decreto 110/2015 modifica la organización de la RAP en la gestión de aceites usados.

En este contexto, se introducen distintos incentivos al objeto de promover la combustión de aceites usados, en particular, en la reforma de la fiscalidad medioambiental -en el ámbito del Impuesto especial sobre la electricidad- y la actualización del régimen retributivo específico de producción de electricidad, a

${ }^{21}$ En Arner y otros $(2005,2006)$ la evaluación de la eficiencia de una subvención y un EMR se efectúa a partir de la relación de las elasticidades de la función de oferta de aceites base regenerados, Es, y la función de demanda de aceites base regenerados, $E d$ :

$$
E_{s} d P_{s}=E_{d} d P_{d}
$$

donde $P_{s}$ es el precio de oferta y $P_{d}$ el precio de demanda.

${ }^{22}$ En 2005, previamente al establecimiento de la RAP en la gestión de aceites usados, el destino a regeneración de los aceites usados era del $65 \%$ de los aceites usados recogidos y el ratio entre el consumo de aceites regenerados y el consumo de lubricantes, del 16,3\%. Desde la entrada en vigor del Real Decreto 679 este porcentaje se sitúa en torno al $20 \%$ del consumo de lubricantes. Por consiguiente, el regulador por el Real Decreto 679 establece un $d r *$ igual al 21,95\% (Arner, 2017a). 
partir de energías renovables, cogeneración y residuos. En consecuencia, surgen distintos SIGs de aceites usados sujetos a RAP (SIGAUS, RAEE, SIGPI, importación de vehículos) que compiten por el aceite usado. El regulador al extender la RAP a otros productos que contienen aceites usados (RAEE) y establecer incentivos a la combustión del aceite usado promueve la competencia entre SIGs, en la gestión del aceite usado, a que hace referencia Fleckinger y Glachant (2010). En este caso, el EMR no está establecido a priori y, de acuerdo con Sigman (1995), el resultado de la evaluación de la eficiencia es indeterminado.

Si se considera la evolución del mercado de lubricantes, debe atenderse a que la RAP contribuyó al diseño más eficiente de los lubricantes, aumentando su vida útil -al sustituir el productor de lubricantes aceites base de primer refino por bases regeneradas- pero tuvo lugar una importante reducción del consumo de lubricantes que, en 2013, es del 35\% (Figura 2). Desde la perspectiva de la oferta, la reducción del consumo de lubricantes se justifica porque la reducción del coste energético de la fabricación de lubricantes, al sustituir el productor de lubricantes aceites base de primer refino por aceites regenerados, conlleva el coste de organización de un sistema de $\mathrm{RAP}^{23}$. A su vez, diversos autores han puesto de manifiesto que los programas de RAP en un contexto de competencia imperfecta, caracterizado por la diferenciación del producto, suponen la reducción del producto (Runkel, 2003, Fleckinger y Glachant, 2010). Actualmente, el mercado de lubricantes en España se caracteriza por una demanda creciente de productos más diferenciados, productos sintéticos y semisintéticos, aceites lubricantes de aceite base de origen no mineral. En consecuencia, una demanda de lubricantes más diferenciada resulta incompatible con el EMR.

\section{CONCLUSIONES}

El Real Decreto 679/2006 estableció lo objetivos de recuperación del 95\% de aceites usados generados en 2006 y de regeneración del 65\% de los aceites usados regenerables en 2008, así como la RAP en la gestión de aceites usados. Los productores de aceites lubricantes constituyeron SIGAUS, fijando la aportación del productor de 0,06€ por kilogramo de aceite comercializado. La evaluación de la eficiencia previamente ha puesto de manifiesto que la RAP en la gestión de aceites usados constituye un EMR y que esta política es más eficiente que una subvención. Asimismo, el precio permiso equivale a la aportación del productor a SIGAUS y el porcentaje de aceite usado de origen desconocido, que SIGAUS asume voluntariamente, del 1,8\%. Desde 2015, el Real Decreto 110/2015 ha modificado la organización de la RAP en la gestión de aceites usados. El nuevo

${ }^{23}$ Se obtienen dos litros de aceite base regenerado de tres litros de aceites usados, mientras que son necesarios cien litros de petróleo para obtener la misma cantidad de aceites base de primer refino (EPA, 1989). 
reparto de responsabilidades ha supuesto que la elasticidad precio de la función de oferta de aceites usados destinados a regeneración disminuya de 1,82 a 0,86 y, a su vez, que aumente la vinculación entre la variable precio y cantidad en la función de demanda, de 0,55 a 0,94. En este caso, el resultado de la evaluación de la eficiencia de la RAP es indeterminado. No obstante, los resultados se presentan asumiendo, en primer lugar, que la estimación por MCOD, aun cuando se acepta desde el punto de vista del ajuste global del modelo, presenta autocorrelación dependiendo del número de retardos considerado. A su vez, en la estimación de la función de demanda el número de ventajas y retardos considerado constituye un caso particular. Por consiguiente, las conclusiones no pueden ser totalmente concluyentes, aunque sí se corresponden con la evolución actual del mercado de aceites usados y el mercado de lubricantes.

Si se consideran los cambios en la gestión del aceite usado, este resultado puede explicarse porque, existiendo competencia entre SIGs por el aceite usado (SIGAUS, RAEE, SIGPI, importación de vehículos) e incentivos a la reutilización del aceite usado como combustible -en el ámbito de la reforma de la Ley de Impuestos Especiales y el régimen específico de producción de electricidad-, el EMR no está establecido a priori. Si se considera el mercado de lubricantes, la evolución creciente de una demanda de lubricantes más diferenciada, principalmente aceites de base no mineral -sintéticos y semisintéticos-, resulta incompatible con el EMR. En este caso, de acuerdo con Fleckinger y Glachant (2010), la evaluación de la eficiencia debe ser mediante un modelo que considere la diferenciación del producto. Asimismo, estos autores y Runkel (2003) señalan que si el mercado no es perfectamente competitivo, la RAP supone la reducción del producto.

En este contexto, desde 2015, se ha recuperado la cantidad de aceite usado destinado a regeneración y combustión. Asimismo, desde 2013 se produce una incipiente recuperación del mercado de lubricantes. El gravamen de $14 €$ por tonelada de aceite lubricante, en el Impuesto sobre hidrocarburos establecido en 2013, constituye un punto de inflexión en el comportamiento del mercado. En 2018, la diferenciación del producto afecta al fuel recuperado de aceites usados o del fuel recuperado de residuos MARPOL tipo c que, de acuerdo con la legislación actual, dejan de tener la condición de residuo. Actualmente, la competencia entre distintas organizaciones de RAP (SIGAUS, RAEE, VFU, SIGPI, importación de vehículos), acompañada de nuevos incentivos a la combustión, tiene por objeto continuar con la recuperación de los distintos tratamientos del aceite usado. A su vez, otros destinos para el aceite usado distintos a la regeneración facilitan la diferenciación de los lubricantes como estrategia de crecimiento en este mercado. 


\section{REFERENCIAS BIBLIOGRÁFICAS}

ANGULO, J.; FERNÁNDEZ DE LAS HERAS, J. y MARTÍN PANTOJA, J.L. (1996). "La regeneración de aceites usados: un proceso viable". Ing. Quím., January, 320, pp. 173-176.

ARNER, A. (2017a). "Evaluación de las políticas de gestión de aceites usados: La responsabilidad ampliada del productor". Annals of Applied Economics, XXXII, pp. 347-360.

ARNER, A. (2017b). "The Spanish used oil market: A vector error correction model". International Journal of Energy Economics and Policy, 7(6), pp. 1-10.

ARNER, A.; BARBERÁN, R. y MUR, J. (2005). "La eficiencia de las políticas para promover la regeneración de aceites usados". II Congreso Ibérico sobre Residuos Generados en la Industria. Madrid: Instituto para la Sostenibilidad de los Recursos.

ARNER, A.; BARBERÁN, R. y MUR, J. (2006). "La eficiencia de las políticas para promover la regeneración de aceites usados". XIII Encuentro de Economía Pública. Almería.

ASELUBE (2018). Memoria de Actividades 2017. Madrid: Asociación Española de Lubricantes. http://www.aselube.es. [Último acceso: Mayo de 2018].

CALCOTT, P. y WALLS, M. (2002). "Waste, Recycling and Design for Environment: Roles for Markets and Policy Instruments". Discussion Paper, Washington, DC: Resources for the Future.

CAMPSA (varios años). Memoria. Compañía Arrendataria del Monopolio de Petróleos. http://www.cnmv.com [Último acceso: Marzo de 2017].

DELEGACIÓN DEL GOBIERNO EN CAMPSA (varios años). Memorias. http://www.cnmv.com. [Último acceso: Marzo de 2017].

DINAN, T. (1993). "Economic efficiency effects of alternative policies for reducing waste disposal". Journal of Environmental Economics and Management, 25, pp. 242-256.

EICHNER, T. y PETHIG, R. (2001). "Product design and efficient management of recycling and waste treatment". Journal of Environmental Economics and Management, 41, pp.109-134.

EPA (1989). How to set up a local program to recycle used oil. Environmental Protection Agency, United States. http://www.epa.gov/osw/conserve/pubs/89039a.pdf. Accessed 1 September 2014.

FLECKINGER, P. y GLACHANT, M. (2010). "The organization of extended producer responsibility in waste policy with product differentiation". Journal of Environmental Economics and Management, 59, pp. 57-66.

FULLERTON, D. y KINNAMAN, T. (1995). "Garbage, recycling, and illicit burning or dumping". Journal of Environmental Economics and Management, 29, pp. 78-91.

FULLERTON, D. y WOLVERTON, A. (2000). "Two generalizations of a deposit-refund system". American Economic Review, 90(2), pp. 238-242.

FULLERTON, D. y WU, W. (1998). "Policies for green design". Journal of Environmental Economics and Management, 36, pp. 131-148.

GÓMEZ-MIÑANA, J.A. (1993). "Los aceites usados. Quién tiene la culpa de que se quemen". Rev. Téc. Medio Ambiente, 34, pp.17-22. 
LLOBET DÍAZ, L. (1995). "Hacia un sistema global de gestión". En CIMAT (ed.):I Conferencia Internacional de Gestión de Residuos RESIDUA 95. Sevilla.

MADDALA, G. y KIM, I. (1999). Unit Roots, Cointegration, and Structural Change. Cambridge: Cambridge University Press.

MASLYUK, S. y DHARMARATNA, D. (2013). "Renewable electricity generation, CO2 emissions and economic growth: Evidence from middle-income countries in Asia". Revista de Estudios de Economía Aplicada, 31(1), pp. 217-244.

MINISTERIO DE MEDIO AMBIENTE (2006). Medio Ambiente en España 2005. Secretaría General Técnica. http://www.magrama.gob.es/es/estadistica/temas/ estadisticas-ambientales/memoria-2005.aspx [último acceso: Octubre de 2016].

MOPTMA (1993). La producción y la gestión de los aceites lubricantes usados en España (1960-1993). Posibilidades de actuación. Madrid: Ministerio de Obras Públicas y Transportes.

NOVALES, A. (2010). Econometría. Madrid: McGraw-Hill.

PALMER, K.; SIGMAN, H.; WALLS. M.; HARRISON, K. y PULLER, S. (1995). "Cost of Reducing Solid Waste: Comparing Deposit-Refunds, Advance Disposal Fees, Recycling Subsidies, and Recycling Rate Standards". Discussion Paper, Washington, DC: Resources for the Future.

PALMER, K. y WALLS, M. (1997). "Optimal policies for solid waste disposal. Taxes, subsidies and standards". Journal of Public Economics, 65, pp. 193-205.

PALMER, K. y WALLS, M. (1999). "Extended Product Responsibility: An Economic Assessment of Alternative Policies". Discussion Paper, Washington, DC: Resources for the Future.

RAMSDEN, D.P. (1995). “Used oil recycling - Quality again”. R'95 Recovery Recycling Re-Integration International Congress. UK.

RUNKEL, M. (2003). "Product durability and extended producer responsibility in solid waste management". Environmental and Resource Economics, 24(2), pp.161-182.

SIGAUS (2013). Memoria de Sostenibilidad 2012. http://www.sigaus.es [Último acceso: Mayo de 2018].

SIGAUS (2016a). Informe a las Administraciones Públicas 2015. Resumen Ejecutivo. http://www.sigaus.es [Último acceso: Marzo de 2017].

SIGAUS (2016b). "Nuevo sistema de financiación 2016". Noticias, no 22. http://www.sigaus.es [Último acceso: Marzo de 2017].

SIGAUS (2018). Informe a las Administraciones Públicas 2017. Resumen Ejecutivo. http://www.sigaus.es [Último acceso: Mayo de 2018].

SIGMAN, H. (1995). "A comparison of public policies for lead recycling". Rand Journal of Economics, 26, pp. 452-478.

TSAI, T.- H.; WU, S.-J. y HWANG, H. (2013). "Waste recycling policies under extended producer responsibility: take-back mandate versus deposit-refund". Global Journal of Economic, 2 (2), pp.1-10.

VOGELSANG, T.J. (1993). Essays on Testing for Nonlinear and Structural Change in Time Series Models. PH.D. Dissertation. Princeton University.

WALLS, M. (2006). "Extended producer responsibility and product design". Discussion Paper. Baltimore: Resources for the Future. 
ZINK, T.; GEYER, R. y STARTZ, R. (2017). "Toward estimating displaced primary production from recycling: a case study of U.S. aluminum". Journal of Industry Ecology, 22(2), pp. 314-326. 
